\title{
NLO QCD corrections to Higgs boson production in association with a top quark pair and a jet
}

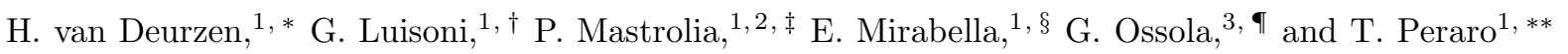 \\ ${ }^{1}$ Max-Planck-Institut für Physik, Föhringer Ring 6, 80805 München, Germany \\ ${ }^{2}$ Dipartimento di Fisica e Astronomia, Università di Padova, and INFN \\ Sezione di Padova, via Marzolo 8, 35131 Padova, Italy \\ ${ }^{3}$ Physics Department, New York City College of Technology, The City \\ University of New York, 300 Jay Street Brooklyn, NY 11201, USA \\ The Graduate School and University Center, The City University of New York, 365 Fifth Avenue, New York, NY 10016, USA
}

\begin{abstract}
We present the calculation of the cross section for Higgs boson production in association with a top quark pair plus one jet, at next-to-leading-order (NLO) accuracy in QCD. All mass dependence is retained without recurring to any approximation. After including the complete NLO QCD corrections, we observe a strong reduction in the scale dependence of the result. We also show distributions for the invariant mass of the top quark pair, with and without the additional jet, and for the transverse momentum and the pseudorapidity of the Higgs boson. Results for the virtual contributions are obtained with a novel reduction approach based on integrand decomposition via Laurent expansion, as implemented in the library NinJA. Cross sections and differential distributions are obtained with an automated setup which combines the GOSAM and SHERPA frameworks.
\end{abstract}

\section{INTRODUCTION}

The evidence of the existence of a new particle of mass between 125 and $126 \mathrm{GeV}$, initially reported about one year ago by the ATLAS and CMS collaborations [1, 2], has been confirmed with very high confidence level by more recent analyses, thus providing more stringent arguments in favor of the validity of the electroweak symmetry breaking mechanism. It is interesting to observe that all the analyses performed so far are in good agreement with the hypothesis that the new particle is the Higgs boson predicted by the Standard Model (SM). Indeed, rates and distributions are compatible with the assumption that the new particle is a scalar that couples to other SM particles with a strength proportional to their mass $[3-5]$. Accurate predictions are necessary and will play a crucial role for the complete determination of the nature of the Higgs boson [6], in particular to shed light on the structure of its couplings to the other particles.

The production rate for a Higgs boson associated with a top-antitop pair $(t \bar{t} H)$ is particularly interesting in this context, since it is directly proportional to the SM Yukawa coupling of the Higgs boson to the top quark. The study of differential observables and distributions will bring information on the coupling structure and on the parity of the Higgs particle [7, 8].

The difficulties related to the analysis of the $t \bar{t} H$ channel are well known. The combined production of three heavy particles requires a large center-of-mass energy for the initial partons, which is strongly suppressed by parton distribution functions. Furthermore, additional difficulties are represented by the presence of various challenging backgrounds and by the complexity of the final state, which make its kinematic reconstruction far from straightforward [9].

At the parton level, the $t \bar{t} H$ production at next-to-
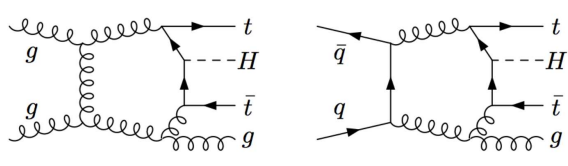

Figure 1. Sample of one-loop diagrams contributing to the NLO corrections to $g g \rightarrow t \bar{t} H g$ and $q \bar{q} \rightarrow t \bar{t} H g$.

leading order (NLO) in QCD has been known for some time [10 14]. More recently, this process has been employed in a number of studies, motivated by the new analyses performed at the LHC $7,9,15]$.

In this letter, we present the complete NLO QCD corrections to the process $p p \rightarrow t \bar{t} H+1$ jet $(t \bar{t} H j)$ at the LHC. Examples of contributing one-loop diagrams are depicted in Fig. 1. We illustrate the outcome of our calculation by showing the total cross section, and a selection of differential distributions.

The goal of the considered calculation is twofold. On the one hand, it is important for the phenomenological analyses at the LHC, in particular for the high- $p_{T}$ region, where the presence of the additional jet can be sensibly relevant. On the other hand, $t \bar{t} H j$ constitutes the first application of a novel reduction algorithm for the evaluation of one-loop amplitudes, which strengthens the performances of the integrand decomposition [16], in particular in the presence of massive particles.

\section{COMPUTATIONAL SET-UP}

In perturbation theory, computations at the NLO accuracy require, aside from the evaluation of leading-order (LO) contributions, the calculation of both virtual and real-emission corrections. The Born and the real emission 
matrix elements are computed using SHERPA [17] and the library AMEgIC [18], which implements the CataniSeymour dipole formalism 19, 20]. SHERPA also performs the integration over the phase space and the analysis. The virtual corrections are generated with the GoSAM package [21], which combines automated diagram generation and algebraic manipulation [22-26] with $d$-dimensional integrand-level reduction techniques 2733]. The master integrals (MIs) are computed using OnELOOP [34]. The code generated by GoSAM is linked to Sherpa by means of the Binoth Les Houches Accord (BLHA) 35] interface, which uses a system of order and contract files and allows for a direct communication between the two codes at running time. The same setup has been recently employed for the computation of NLO QCD corrections to $p p \rightarrow H j j$ [36] and $p p \rightarrow H j j j$ [37] (for the latter, in combination with MadDipole/MadEvent 38 40]) and also for the analysis of the $t \bar{t}$ forward-backward asymmetry [41].

For $t \bar{t} H j$ production, the basic partonic processes identified by the SHERPA-GoSAm contract file are:

$$
q \bar{q} \rightarrow t \bar{t} H g, \quad g g \rightarrow t \bar{t} H g
$$

while the remaining subprocesses can be obtained by proper crossings. The ultraviolet, the infrared, and the collinear singularities are regularized using dimensional reduction. The renormalization conditions are fixed along the lines of [11, 13], where the top mass is renormalized on-shell, while the strong coupling is renormalized in the $\overline{\mathrm{MS}}$ scheme, decoupling the top quark from the running. In the case of $\mathrm{LO}$ [NLO] contributions, we describe the running of the strong coupling constant with one-loop [two-loop] accuracy. The wave functions of the gluon and of the quarks are renormalized on-shell, i.e. the corresponding renormalization constants cancel the external self-energy corrections exactly.

The virtual amplitudes of $t \bar{t} H j$ have been decomposed in terms of MIs using for the first time the integrand reduction via Laurent expansion [16], implemented in the $\mathrm{C}++$ library Ninja. This new algorithm exploits the complete knowledge of the analytic expression of the integrand and of the residues at the multiple cut to ameliorate the determination of the coefficients of the MIs with respect to the canonical integrand reduction 27]. Elaborating on the techniques introduced in 42 44], the series expansion combined with the integrand decomposition lowers the computational load and improves the accuracy of the results. Within this new algorithm, the sampling of the numerators and the subtractions of the higher-point residues, characterizing the triangular system-solving approach of the original integrand-reduction procedure, are avoided. Instead, the series expansion allows for a diagonal system-solving strategy, where the polynomial subtractions of the residues, when needed, are replaced by universal correction terms which have to be added to the

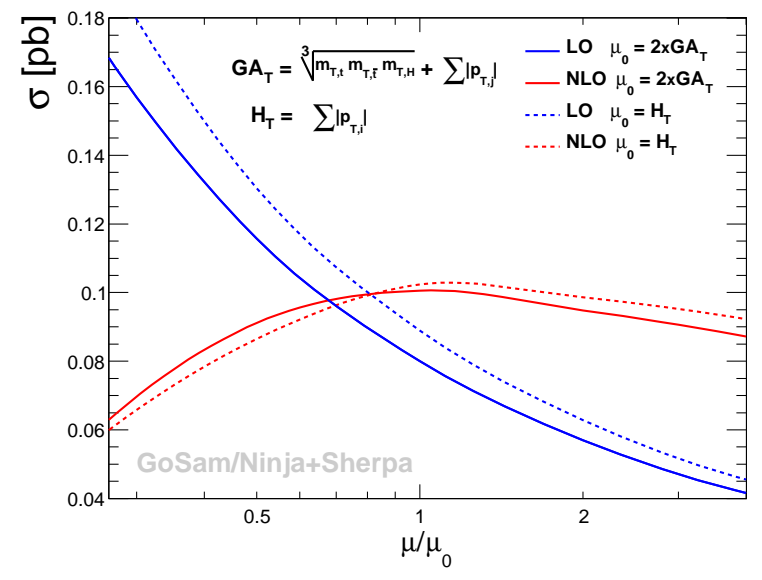

Figure 2. Scale dependence of the total cross section at LO and NLO.

coefficients of the Laurent series. These universal corrections, required only for the determination of the coefficients of 2-point and 1-point MIs, are obtained, once and for all, from the expansions of the generic polynomial forms of the residues at the triple and double cuts.

The NinJa library, which has been interfaced to GoSAM, implements the integrand reduction via Laurent expansion using a semi-analytic algorithm. The coefficients of the Laurent expansion of a generic integrand are efficiently computed by performing a polynomial division between the numerator and the set of uncut denominators [16].

The calculation of the NLO virtual corrections performed with NinJa has been checked using the independent reduction algorithm implemented in the library SAMURAI 32]. We verified the agreement of the virtual corrections obtained with the two reduction procedures in ten thousand phase-space points. The values of double and the single poles, for each individual subprocess, conform to the universal singular behavior of dimensionally regulated one-loop amplitudes [45]. Our results fulfill gauge invariance, verified through the vanishing of the amplitudes when substituting the polarization vector of one or more gluons with the corresponding momentum.

The NinJa reduction algorithm proved to be numerically more efficient and stable. In fact, for the highly non-trivial process under consideration, only a small set of phase-space points, of the order of few per mill, were detected as unstable. All these points have been recovered using the tensorial reduction provided by Golem95 [46, 47], thus avoiding the necessity of higher precision routines, which are extremely time consuming.

The time required for the computation of the full colorand helicity-summed amplitudes in one phase-space point is about 2.5 seconds. The numerical values of the oneloop amplitudes for the two partonic processes listed in 


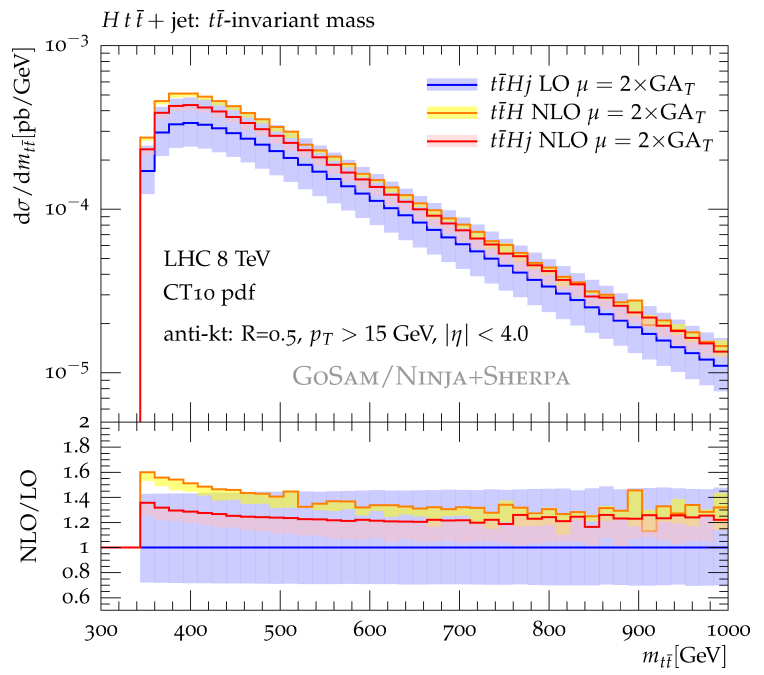

Figure 3. Invariant mass distributions of the $t \bar{t}$-pairs for $t \bar{t} H$ and $t \bar{t} H j$ at NLO relative to the $t \bar{t} H j$ at LO for $\mu=2 \times \mathrm{GA}_{T}$.

Eq. (11) in a non-exceptional phase-space point are collected in the Appendix.

In view of the later comparison between the processes $p p \rightarrow t \bar{t} H$ and $p p \rightarrow t \bar{t} H j$ at NLO QCD accuracy, we also used the GoSAm/NinJa+ShERPa framework to compute the cross section for $t \bar{t} H$ production. We found excellent agreement with the results presented in Refs. [7, 48].

\section{NUMERICAL RESULTS}

In the following, we present results for the integrated cross section for a center-of-mass energy of $8 \mathrm{TeV}$. The mass of the Higgs boson is set to $m_{H}=126 \mathrm{GeV}$ and the top quark mass is set to $m_{t}=172.5 \mathrm{GeV}$. The parameters of the electroweak sector are fixed by setting $M_{W}=80.419 \mathrm{GeV}, M_{Z}=91.1876 \mathrm{GeV}$ and $\alpha_{E W}^{-1}=132.50698$.

To cluster the jets we use the antikt-algorithm implemented in FASTJET [49 51] with radius $R=0.5$, a minimum transverse momentum of $p_{T, j e t}>15 \mathrm{GeV}$ and pseudorapidity $|\eta|<4.0$. The LO cross sections are computed with the LO parton-distribution functions cteq6L1 [52], whereas at NLO we use CT10 [53].

In order to study the scale dependence of the total cross section, we employ two different choices of the renormalization and factorization scales $\mu_{R}=\mu_{F}=\mu_{0}$, namely $\mu_{0}=H_{T}$ and $\mu_{0}=2 \times \mathrm{GA}_{T}$ with

$$
\begin{aligned}
H_{T} & =\sum_{\substack{\text { final } \\
\text { states } f}}\left|p_{T, f}\right| \\
\mathrm{GA}_{T} & =\sqrt[3]{m_{T, H} m_{T, t} m_{T, \bar{t}}}+\sum_{\text {jets } j}\left|p_{T, j}\right| .
\end{aligned}
$$

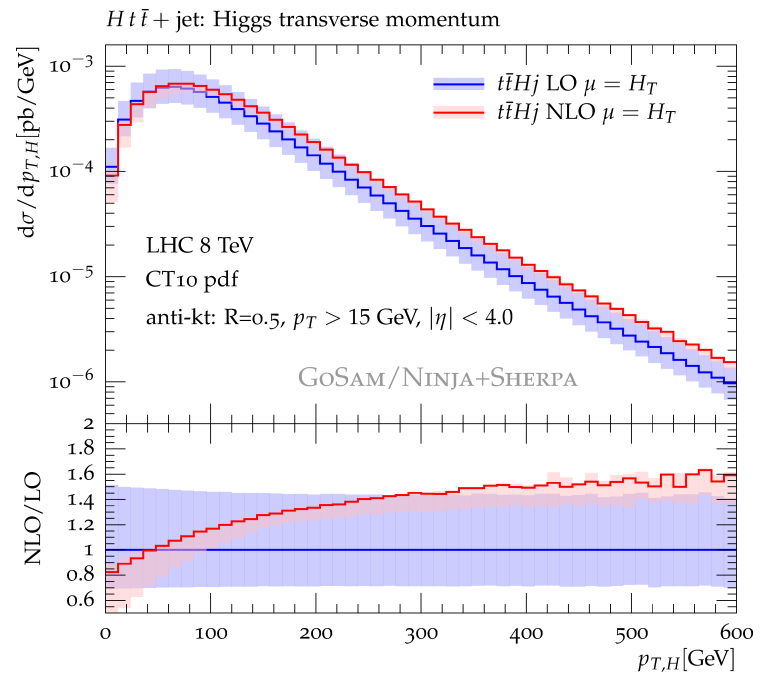

Figure 4. Transverse momentum distribution of the Higgs boson at LO and NLO for $\mu=H_{T}$.

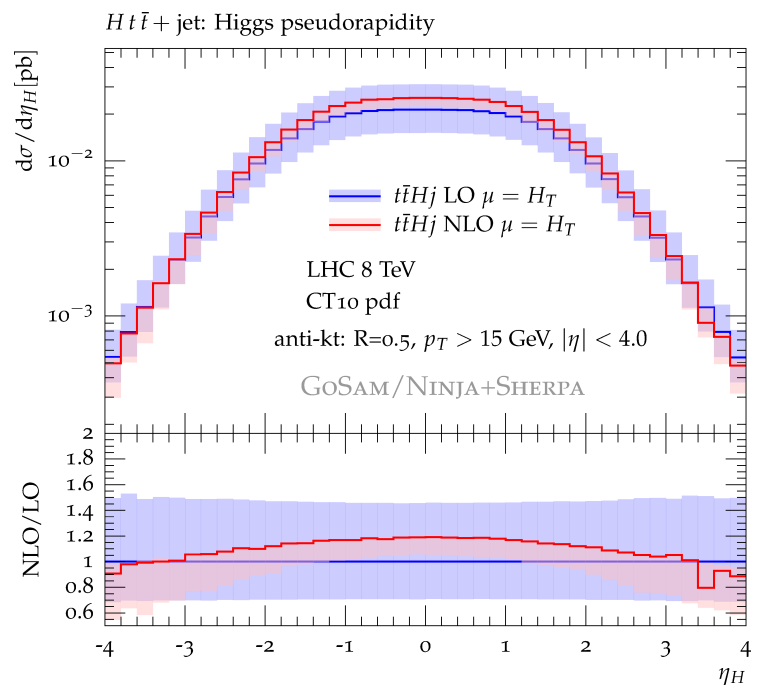

Figure 5. Pseudorapidity $\eta$ of the Higgs boson at LO and NLO accuracy for $\mu=H_{T}$.

Within this setup, for the two scale choices, we obtain the total LO and NLO cross sections reported in Table【.

\begin{tabular}{ccc}
\hline \hline CENTRAL SCALE & $\sigma_{L O}[\mathrm{fb}]$ & $\sigma_{N L O}[\mathrm{fb}]$ \\
\hline $2 \times \mathrm{GA}_{T}$ & $80.03_{-23.02}^{+35.64}$ & $100.6_{-9.43}^{+0.00}$ \\
$H_{T}$ & $88.93_{-26.13}^{+41.41}$ & $102.3_{-15.82}^{+0.00}$ \\
\hline \hline
\end{tabular}

Table I. Total cross section for $t \bar{t} H j$ for different choices of the central scale at LO and NLO.

The scale dependence of the total cross section, depicted in Fig. 2. is strongly reduced by the inclusion of 
the NLO contributions. It is worthwhile to notice that both choices for the central value of the scale provide an adequate description, being close to the physical scale of the process.

In Fig. 3, we compare the distributions for the invariant mass of the top quark pair in $p p \rightarrow t \bar{t} H j$ at LO and NLO with the NLO curve for $p p \rightarrow t \bar{t} H$. For $t \bar{t} H j$, going from LO to NLO accuracy, we observe an increase in the distribution by $20-35 \%$ over the full kinematical range. On the other hand, when comparing the NLO $t \bar{t} H$ prediction with the NLO $t \bar{t} H j$ curve, the cross section decreases due to the presence of the additional jet which takes away energy from the $t \bar{t}$ system. This is particularly evident near the $t \bar{t}$ production threshold, while for high values of the $t \bar{t}$ invariant mass the two NLO curves get closer. The scale for this comparison is set to $\mu=2 \times \mathrm{GA}_{T}$.

In Fig. 4 and Fig. 5 we display the distributions of the transverse momentum $p_{T}$ and the pseudorapidity $\eta$ of the Higgs boson, respectively. Each plot contains the distributions at LO and NLO accuracy, for a value of the scale set to $\mu=H_{T}$. The NLO corrections are particularly important for high values of the $p_{T}$, which are the kinematical regions involved in the boosted analyses [15, 54].

These distributions show the potential of the framework obtained combining GoSAm/NINJA with SHERPA, which can be successfully used to compute NLO predictions for multi-leg processes involving massive particles. Moreover they shed some light on the impact of further jet activity in $p p \rightarrow t \bar{t} H$, one of the most important processes for the direct determination of the coupling of the Higgs boson to fermions. The NLO QCD corrections reduce the scale uncertainty and their numerical impact can be sizable. Therefore they could be helpful for an accurate simulation of the signal in the experimental searches looking for Higgs production in association with a top-antitop pair at the LHC.

Acknowledgments - We thank all the other members of the GoSAm project for collaboration on the common development of the code. We also thank Jan Winter for interesting discussions. The work of H.v.D., G.L., P.M., and T.P. was supported by the Alexander von Humboldt Foundation, in the framework of the Sofja Kovaleskaja Award Project "Advanced Mathematical Methods for Particle Physics", endowed by the German Federal Ministry of Education and Research. G.O. was supported in part by the National Science Foundation under Grant PHY-1068550. Computing resources were provided by the CTP cluster of the New York City College of Technology.

\section{Benchmark phase-space point}

In this appendix we collect numerical results for the renormalized virtual contributions to the processes (1), in correspondence to the phase-space point in Table [I] The results are collected in Table $\amalg$ and are computed using dimensional reduction. The coefficients $a_{i}$ are defined as follows:

$$
\frac{a_{-2}}{\epsilon^{2}}+\frac{a_{-1}}{\epsilon}+a_{0} \equiv \frac{2 \mathfrak{R e}\left\{\mathcal{M}^{\text {tree-level* }} \mathcal{M}^{\text {one-loop }}\right\}}{\left(\alpha_{s} / 2 \pi\right)\left|\mathcal{M}^{\text {tree-level }}\right|^{2}} .
$$

The reconstruction of the renormalized pole can be checked against the value of $a_{-1}$ and $a_{-2}$ obtained by the universal singular behavior of the dimensionally regularized one-loop amplitudes [45], while the precision of the finite parts is estimated by re-evaluating the amplitudes for a set of momenta rotated by an arbitrary angle about the axis of collision.

* hdeurzen@mpp.mpg.de

† luisonig@mpp.mpg.de

‡ pierpaolo.mastrolia@cern.ch

$\S$ mirabell@mpp.mpg.de

ฯ gossola@citytech.cuny.edu

** peraro@mpp.mpg.de

[1] G. Aad et al. (ATLAS Collaboration), Phys.Lett. B716, 1 (2012), 1207.7214.

[2] S. Chatrchyan et al. (CMS Collaboration), Phys.Lett. B716, 30 (2012), 1207.7235.

[3] CMS-PAS-HIG-13-005 (2013).

[4] G. Aad et al. (ATLAS Collaboration) (2013), 1307.1427.

[5] T. Aaltonen et al. (CDF Collaboration, D0 Collaboration) (2013), 1303.6346.

[6] S. Heinemeyer et al. (The LHC Higgs Cross Section Working Group) (2013), 1307.1347.

[7] R. Frederix, S. Frixione, V. Hirschi, F. Maltoni, R. Pittau, et al., Phys.Lett. B701, 427 (2011), 1104.5613.

[8] C. Degrande, J. Gerard, C. Grojean, F. Maltoni, and G. Servant, JHEP 1207, 036 (2012), 1205.1065.

[9] P. Artoisenet, P. de Aquino, F. Maltoni, and O. Mattelaer (2013), 1304.6414.

[10] W. Beenakker, S. Dittmaier, M. Kramer, B. Plumper, M. Spira, et al., Phys.Rev.Lett. 87, 201805 (2001), hep$\mathrm{ph} / 0107081$.

[11] W. Beenakker, S. Dittmaier, M. Kramer, B. Plumper, M. Spira, et al., Nucl.Phys. B653, 151 (2003), hep$\mathrm{ph} / 0211352$.

[12] S. Dawson, L. Orr, L. Reina, and D. Wackeroth, Phys.Rev. D67, 071503 (2003), hep-ph/0211438.

[13] S. Dawson, C. Jackson, L. Orr, L. Reina, and D. Wackeroth, Phys.Rev. D68, 034022 (2003), hepph/0305087.

[14] S. Dittmaier, . Kramer, Michael, and M. Spira, Phys.Rev. D70, 074010 (2004), hep-ph/0309204.

[15] T. Plehn, G. P. Salam, and M. Spannowsky, Phys.Rev.Lett. 104, 111801 (2010), 0910.5472.

[16] P. Mastrolia, E. Mirabella, and T. Peraro, JHEP 1206 , 095 (2012), 1203.0291.

[17] T. Gleisberg, S. Hoeche, F. Krauss, M. Schonherr, S. Schumann, et al., JHEP 0902, 007 (2009), 0811.4622.

[18] F. Krauss, R. Kuhn, and G. Soff, JHEP 0202, 044 (2002), hep-ph/0109036. 


\begin{tabular}{ccccc}
\hline \hline particle & $E$ & $p_{x}$ & $p_{y}$ & $p_{z}$ \\
\hline$p_{1}$ & 250.00000000000000 & 0.0000000000000000 & 0.0000000000000000 & 250.00000000000000 \\
$p_{2}$ & 250.00000000000000 & 0.0000000000000000 & 0.0000000000000000 & -250.00000000000000 \\
$p_{3}$ & 177.22342332868467 & -31.917865771774753 & -19.543909461587205 & -15.848571666570733 \\
$p_{4}$ & 174.89951284907735 & 13.440699620020803 & 24.1748998117950033 & -8.2771667589629576 \\
$p_{5}$ & 126.37478917634435 & 6.8355633672742222 & -3.2652801590882752 & 6.0992096455298030 \\
$p_{6}$ & 21.502274645893632 & 11.641602784479652 & -1.3657084972745175 & 18.026528780003872 \\
\hline \hline
\end{tabular}

Table II. Benchmark phase-space point for $t \bar{t} H j$ production

\begin{tabular}{lrr}
\hline \hline & $q \bar{q} \rightarrow t \bar{t} H g$ & $g g \rightarrow t \bar{t} H g$ \\
\hline$a_{0}$ & $\underline{-80.40233474207548}$ & -45.69793352903498 \\
$a_{-1}$ & $\frac{-32.69029102067917}{-35.9217497445} 3633$ \\
$a_{-2}$ & $\underline{-5.666666666667901}$ & $\underline{-9.00000000000} 6723$ \\
\hline \hline
\end{tabular}

Table III. Numerical results for the two subprocesses listed in Eq. (1) evaluated at the phase-space point of Table II for a scale $\mu=2 p_{1} \cdot p_{2}=500 \mathrm{GeV}$. The accuracy of the result is indicated by the underlined digits.

[19] S. Catani, S. Dittmaier, M. H. Seymour, and Z. Trocsanyi, Nucl.Phys. B627, 189 (2002), hep-ph/0201036.

[20] T. Gleisberg and F. Krauss, Eur.Phys.J. C53, 501 (2008), 0709.2881.

[21] G. Cullen, N. Greiner, G. Heinrich, G. Luisoni, P. Mastrolia, et al., Eur.Phys.J. C72, 1889 (2012), 1111.2034.

[22] P. Nogueira, J.Comput.Phys. 105, 279 (1993).

[23] J. A. M. Vermaseren (2000), math-ph/0010025.

[24] T. Reiter, Comput.Phys.Commun. 181, 1301 (2010), 0907.3714.

[25] G. Cullen, M. Koch-Janusz, and T. Reiter, Comput.Phys.Commun. 182, 2368 (2011), 1008.0803.

[26] J. Kuipers, T. Ueda, J. Vermaseren, and J. Vollinga, Comput.Phys.Commun. 184, 1453 (2013), 1203.6543.

[27] G. Ossola, C. G. Papadopoulos, and R. Pittau, Nucl.Phys. B763, 147 (2007), hep-ph/0609007.

[28] G. Ossola, C. G. Papadopoulos, and R. Pittau, JHEP 0707, 085 (2007), 0704.1271.

[29] R. K. Ellis, W. T. Giele, and Z. Kunszt, JHEP 03, 003 (2008), 0708.2398.

[30] G. Ossola, C. G. Papadopoulos, and R. Pittau, JHEP 0805, 004 (2008), 0802.1876.

[31] P. Mastrolia, G. Ossola, C. Papadopoulos, and R. Pittau, JHEP 0806, 030 (2008), 0803.3964.

[32] P. Mastrolia, G. Ossola, T. Reiter, and F. Tramontano, JHEP 1008, 080 (2010), 1006.0710.

[33] G. Heinrich, G. Ossola, T. Reiter, and F. Tramontano, JHEP 1010, 105 (2010), 1008.2441.

[34] A. van Hameren, Comput.Phys.Commun. 182, 2427 (2011), 1007.4716.

[35] T. Binoth, F. Boudjema, G. Dissertori, A. Lazopoulos, A. Denner, et al., Comput.Phys.Commun. 181, 1612
(2010), 1001.1307.

[36] H. van Deurzen, N. Greiner, G. Luisoni, P. Mastrolia, E. Mirabella, et al., Phys.Lett. B721, 74 (2013), 1301.0493.

[37] G. Cullen, H. van Deurzen, N. Greiner, G. Luisoni, P. Mastrolia, et al., Phys.Rev.Lett. 111, 131801 (2013), 1307.4737.

[38] F. Maltoni and T. Stelzer, JHEP 0302, 027 (2003), hep$\mathrm{ph} / 0208156$.

[39] R. Frederix, T. Gehrmann, and N. Greiner, JHEP 0809 , 122 (2008), 0808.2128.

[40] R. Frederix, T. Gehrmann, and N. Greiner, JHEP 1006, 086 (2010), 1004.2905.

[41] S. Hoeche, J. Huang, G. Luisoni, M. Schoenherr, and J. Winter, Phys.Rev. D88, 014040 (2013), 1306.2703.

[42] D. Forde, Phys. Rev. D75, 125019 (2007), 0704.1835.

[43] W. B. Kilgore (2007), 0711.5015.

[44] S. D. Badger, JHEP 01, 049 (2009), 0806.4600.

[45] S. Catani, S. Dittmaier, and Z. Trocsanyi, Phys.Lett. B500, 149 (2001), hep-ph/0011222.

[46] T. Binoth, J.-P. Guillet, G. Heinrich, E. Pilon, and T. Reiter, Comput.Phys.Commun. 180, 2317 (2009), 0810.0992 .

[47] G. Cullen, J. Guillet, G. Heinrich, T. Kleinschmidt, E. Pilon, et al., Comput.Phys.Commun. 182, 2276 (2011), 1101.5595.

[48] V. Hirschi, R. Frederix, S. Frixione, M. V. Garzelli, F. Maltoni, et al., JHEP 1105, 044 (2011), 1103.0621.

[49] M. Cacciari and G. P. Salam, Phys.Lett. B641, 57 (2006), hep-ph/0512210.

[50] M. Cacciari, G. P. Salam, and G. Soyez, JHEP 0804, 063 (2008), 0802.1189.

[51] M. Cacciari, G. P. Salam, and G. Soyez, Eur.Phys.J. C72, 1896 (2012), 1111.6097.

[52] J. Pumplin, D. Stump, J. Huston, H. Lai, P. M. Nadolsky, et al., JHEP 0207, 012 (2002), hep-ph/0201195.

[53] H.-L. Lai, M. Guzzi, J. Huston, Z. Li, P. M. Nadolsky, et al., Phys.Rev. D82, 074024 (2010), 1007.2241.

[54] J. M. Butterworth, A. R. Davison, M. Rubin, and G. P. Salam, Phys.Rev.Lett. 100, 242001 (2008), 0802.2470. 